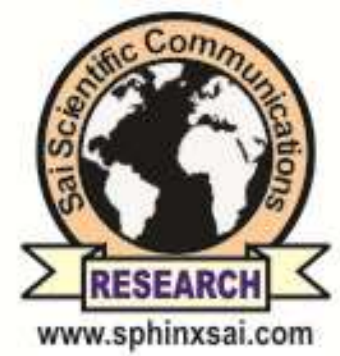

International Journal of PharmTech Research CODEN (USA): IJPRIF, ISSN: 0974-4304, ISSN(Online): 2455-9563

Vol.13, No.02, pp 110-122,

2020

\title{
Evaluation of Neuroprotective and In-vitro Anti-oxidant Activity Isolated Hexane and Ethyl Acetate Fraction from Methanolic Extract of Biophytum reinwardtii
}

\author{
Bandaru Nagaraju ${ }^{1 *}$, A.Ramu ${ }^{2}$, S.Vidhyadhara ${ }^{3}$ \\ ${ }^{1}$ Research scholar, Department of Pharmaceutical Sciences, Chebrolu Hanumaiah \\ Institute of Pharmaceutical Sciences, Guntur, India \\ ${ }^{2,3}$ Professor, Department of Pharmaceutics, Chebrolu Hanumaiah Institute of \\ Pharmaceutical Sciences, Guntur, India
}

\begin{abstract}
Nature is the best source of complementary and alternative medicine. The plant Biophytum reinwardtii has been used traditionally in pain, inflammatory and oxidative stress related disorders. In this consequence, fraction of methanolic extract of Biophytum reinwardtii was selected to explore the ability of this plant to enhance cognitive function, brain antioxidant enzymes and anti-acetyl cholinesterase activity which can be used for the treatment of oxidative stress related disorders like Alzheimer's disease (AD). The purpose of this study was to investigate the neuroprotective effect of HEMBR on learning and memory impairment in scopolamine-induced rats of dementia and oxidative stress. Treatment with HEMBR (i.e., 50 and $100 \mathrm{mg} / \mathrm{kg}$ b.w.) was investigated in scopolamine-treated Swiss albino male rats for 7 days and its neuroprotective effects were examined using Elevated Plus Maze (EPM) test, Passive Avoidance (PA) test and, Morris Water Maze (MWM) test as well as level of antioxidant enzymes such as catalase (CAT), reduced glutathione (GSH) and acetylcholinesterase (AChE) activity in rat brain tissue homogenates. The present study demonstrates that HEMBR showed the neuroprotective effect by improving cognitive functions and reduces oxidative stress by increasing the level of brain antioxidant enzymes as well as decreasing acetylcholinesterase activity. Therefore, this plant extract faction can be used for enhancing learning, memory, antioxidant potentiality and anti-acetylcholinesterase activity in neurodegenerative disorders like AD.

Keywords : Dementia, Scopalamine, Cholinesterase, memory, HEMBR.
\end{abstract}

Introduction:

Alzheimer's disease (AD) is the most common form of dementia considered as progressive, neurodegenerative disease characterized by the presence of senile plaques rich in insoluble aggregate of betaamyloid and neurofibrillary tangles in the brain. ${ }^{1}$ Factors such as decreased acetylcholine level, oxidative stress, and hypercholesterolemia have been reported in memory decline. ${ }^{2}$ Currently, 30 million people are affected

Bandaru Nagaraju et al /International Journal of PharmTech Research, 2020,13(2): 110-122.

DOI= http://dx.doi.org/10.20902/lJPTR.2019.130214 
worldwide by dementia with 4.6 million cases annually as per AD International analysis. ${ }^{3}$ Many acetyl cholinesterase inhibitor drugs are available in the market to treat, but they have modest benefits and associated with side effects; hence, different drugs are aiming at diverse targets which are expected to act better than single targeting agents to treat $\mathrm{AD} .{ }^{4}$ Donepezil, a reversible non-competitive cholinesterase enzyme inhibitor considered as first-line treatment in AD which is evident from recent report of "cholinergic anti-inflammation" pathway, is responsible for neuroprotective effect; ${ }^{5}$ however, its beneficial effect on memory is temporarily for 36 weeks and the progression of $\mathrm{AD}$ cannot be reversed or modified for long time; ${ }^{6}$ hence, noncholinergic therapies are also needed to overcome these problems.

Scopolamine, a cholinergic antagonist, impairs learning and memory which is a characteristic manifestation of dementia; ${ }^{7}$ hence, in this study, scopolamine-induced amnesia was used as an experimental model.

Biophytum reinwardtii(Family - Oxalidaceae), commonly known as "Pulicenta" in south India. The present work attempts to isolation of fractions from methanolic extract and evaluate neuroprotective activity of fraction in scopolamine induced dementia

\section{Materials and Methods}

\section{General experimental procedures}

Ready-made TLC plates loaded with silica gel 60 F254 (Merck, Darmstadt, Germany) were Applied for analytical purposes. 1D (1H and 13C NMR; chemical shifts in ppm) and 2D NMR spectra were measured using Bruker AVANCE HD III NMR spectrometer (Switzerland) using deuterated solvents (Sigma Aldrich, Germany).

\section{Plant material}

Biophytum reinwardtii whole plant was collected from forest area of Rampachowdavaram, East Godavari and authenticated by Dr.P.Prasanna Kumari Department of Botany, D.N.R College, Bhimavaram A voucher specimen was kept at Department of Pharmacology, Shri Vishnu College of Pharmacy.

\section{Extraction and isolation of the fractions}

\section{Preparation of plant extract:}

The Whole plant were dried under shade at room temperature The shade dried, coarsely powdered roots $(500 \mathrm{~g})$ was successively extracted with petroleum ether $(60-80 \mathrm{oC})$ for 7 days to remove fatty matter. The defatted marc was then subjected to soxhlet extraction with $95 \%$ methanol to obtain methanolic extract. The methanolic extract was evaporated under reduced pressure at low temperature $(30 \mathrm{oC})$ to dryness and brownish yellow colour extracts of Biophytum reinwardtii was obtained. ${ }^{8}$

\section{Separation of active compounds by column chromatography:}

Column-chromatography A cylinder shaped glass column containing stationary phase (silica gel) is encountered slowly from the top with a liquid solvent (mobile phase) that flows down the column with the help gravity or external pressure applied. This technique is used for the purification of compounds from a mixture. Once the column is ready, the sample is loaded inside the top of the column. The mobile solvent is then allowed to flow down through the column. The compounds in mixture have different interactions ability with stationary phase (silica gel), and mobile phase, thereby will flow along the mobile phase at different time intervals or degrees. In this way, the separation of compounds from the mixture is achieved. The individual compounds are collected as fractions and analyzed further for structure elucidation. ${ }^{9}$

\section{Protocol}

Isolation and purification of bioactive compounds from plant samples

1. A suitable size long cylindrical glass column (based on the amount of the sample) should be stand firm on a column-chromatography stand. 
2. Completely dried plant extract sample should be mixed with silica gel to make a fine powdered form for easy distribution of sample in already packed silica gel column.

3. Sample powdered mass should be placed on the top of the pre-packed silica column and sample should be covered with a layer of cotton.

4. Then solvents of different polarities were passed through column at uniform rate under gravity to fractionate the sample extract.

5. Each fraction was collected separately in a test tube and numbered consecutively for further analysis on thin layer chromatography.

6. Thin layer chromatography (TLC) provides partial separation of both organic and inorganic materials using thin-layered chromatographic plates especially useful for checking the purity of fractions.

7. Each fraction is applied on activated TLC plates with the help of capillary tube at a $1 / 2$ inch apart from the lower edge of TLC plate, and plate is kept in a developing chamber containing suitable solvent system for specific time until the developing solvent reaches top of the upper edge of TLC plate.

8. Plate is taken out from developing chamber, dried and solvent front is marked by lead pencil. Compound bands/spots visualized on TLC chromatoplate can be detected by visual detection, under UV light (254 nm), in iodine chamber and by using spray reagent (vanillin-sulfuric acid) for the presence of specific compounds.

Table:1: Gradient solvent system used in column chromatography

\begin{tabular}{|l|l|l|}
\hline $\begin{array}{l}\text { Gradient solvent system used in column chromatography for the isolation of fractions in } \\
\text { Biophytum reinwardtii whole plant }\end{array}$ \\
\hline Solvent system & Ratio & Fraction \\
\hline Hexane & $100 \%$ & - \\
\hline Hexane : Ethyl acetate & $\mathbf{9 5 : 5}$ & A \\
\hline Hexane : Ethyl acetate & $70: 30$ & B \\
\hline Hexane : Ethyl acetate & $50: 50$ & C \\
\hline Only ethyl acetate & $100 \%$ & D \\
\hline Only methanol & $100 \%$ & E \\
\hline
\end{tabular}

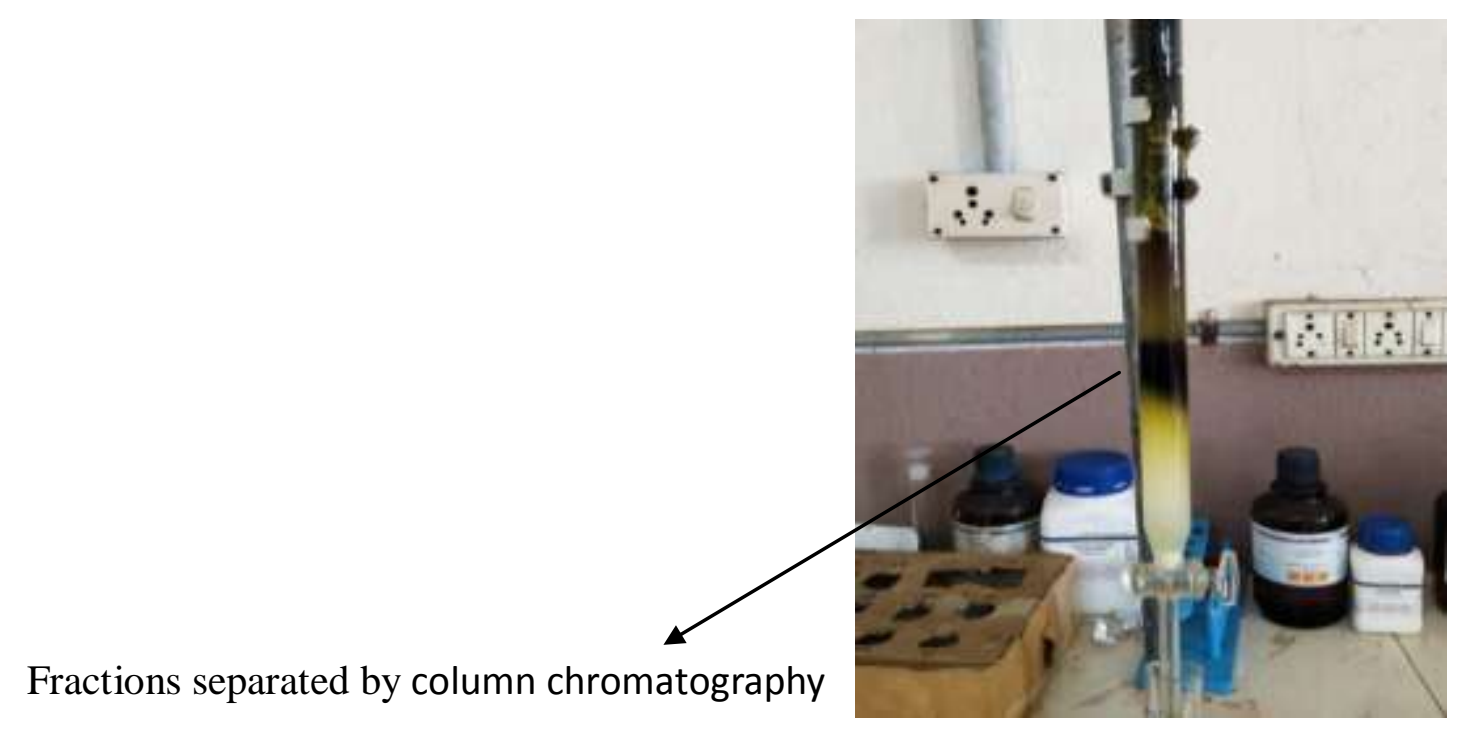

Figure: 1: Separation of fractions by column chromatography

\section{Preliminary Phytochemical Screening}

Different fractions were subjected to preliminary phytochemical for the detection of various constituents. 


\section{Acute toxicity studies}

Acute toxicity studies were performed according to organization for economic co-operation and development OECD guidelines 429. Animals were divided in groups $(n=4)$ and fasted for $4 \mathrm{~h}$ with free access to water only. The Hexane and Ethyl Acetate fraction A was administered orally in doses of 5, 50,200 and 2000 $\mathrm{mg} / \mathrm{kg}$ to different groups of mice and observed over $24 \mathrm{hr}$. for mortality and physical/ behavioural changes. ${ }^{10}$

\section{Determination of in vitro Antioxidant activity Free radical scavenging (DPPH) assay}

The free radical scavenging activity of The Hexane and Ethyl Acetate fraction A was measured in vitro by 1,1-diphenyl-2-picryl-hydrazyl (DPPH) assay using the method of Blois (1958). About 0.3mM solution of DPPH in $100 \%$ ethanol was prepared and $1 \mathrm{ml}$ of this solution was added to $3 \mathrm{ml}$ of theFraction dissolved in ethanol at different concentrations $\left(10-50 \_\mathrm{g} / \mathrm{ml}\right)$. The mixture was shaken and allowed to stand at room temperature for $30 \mathrm{~min}$ and the absorbance was measured at $517 \mathrm{~nm}$ using a spectrophotometer. The IC50 value of the crude extract was compared with that of ascorbic acid, which was used as the standard. ${ }^{11}$

\section{Procedure for Scopolamine induced Dementia}

\section{Animals used in the study:}

Healthy adult albino Wistar rats weighing 200-250grams of Wistar rats were selected for the study. Animals were housed in appropriate cages in uniform hygienic conditions and fed with standard pellet diet (Amrul Laboratory ANIMAL Diet) and water ad Libitum. Animal studies had approval of IACE committee for the purpose of control and supervision of experiments on animals (CPCSEA).

\section{Experimental design}

Animals were classified into five groups (6 rats each). Treatments were given p.o. for seven successive days. One hour after the last dose of test agents, all animals were i.p. injected with scopolamine hydrobromide $(5 \mathrm{mg} / \mathrm{kg}$ ) except the first group (control group). Animals were treated according to the following scheme: groups I and II received 1\% Tween 80; meanwhile groups III and IV received donepezil hydrochloride $(2.5 \mathrm{mg} / \mathrm{kg}$ ) or Fraction A (H:EA) $(50$ and $100 \mathrm{mg} / \mathrm{kg})$, respectively. Spatial learinig, active avoid response and locomotors activity tests were conducted $30 \mathrm{~min}$ after scopolamine injection. ${ }^{12-14}$ immediately after performing the behavioral tests, rats were sacrificed by decapitation, brains were rapidly isolated. Each brain was dissected through the midline into two hemispheres. Each hemisphere was weighed and homogenized in ice-cold $50 \mathrm{mM}$ phosphate buffer $(\mathrm{pH}$ 7.4) to prepare $10 \%$ homogenate that was used for the estimation cholinesterase and catalase and glutathione .Finally, brains of 2-3 rats from each group were preserved in $10 \%$ formalin and kept for histopathologic examination.

Total 30 rats were taken, each group contains 6rats.

$\begin{array}{ll}\text { Group I } & \text { : Received normal saline } \\ \text { Group II } & \text { : Scopolamine } 5 \mathrm{mg} / \mathrm{kg} \text { (i.p.) for } 7 \text { days. } \\ \text { Group III } & \text { : Scopolamine } 5 \mathrm{mg} / \mathrm{kg} \text { (i.p.). Donepezil }(2 \mathrm{mg} / \mathrm{kg} \text { ) for } 7 \text { days. } \\ \text { Group IV } & \text { : Scopolamine } 5 \mathrm{mg} / \mathrm{kg} \text { (i.p.). Fraction A } 50 \mathrm{mg} / \mathrm{kg} \text { for } 7 \text { days } \\ \text { Group V } & \text { : Scopolamine } 5 \mathrm{mg} / \mathrm{kg} \text { (i.p.). Fraction A } 100 \mathrm{mg} / \mathrm{kg} \text { for } 7 \text { days }\end{array}$

\section{Estimation of Acetyl cholinesterase levels:}

The assay is based on measurement of the change in absorbance at $412 \mathrm{~nm}$. The assay uses the thiol ester acetylthiocholine instead of the oxy ester acetylcholine. AChE hydrolyses the acetyl-thiocholine to produce thiocholine and acetate. ${ }^{15}$ The thiocholine in turn reduces the dithiobis-nitrobenzoic acid (DTNB) liberating nitro-benzoate, which absorbs at $412 \mathrm{~nm}$.

\section{Estimation of Catalase activity:}

Catalases are enzymes that catalyze the conversion of hydrogen peroxide to water and oxygen, using either an iron or manganese cofactor. This protein is localized to peroxisomes in most eukaryotic cells. Catalase is an unusual enzyme since, although hydrogen peroxide is its only substrate and follows a Ping-Pong 
mechanism. ${ }^{16-17}$ Its cofactor is oxidised by one molecule of hydrogen peroxide and then regenerated by transferring the bound oxygen to a second molecule of substrate. Despite its apparent importance in hydrogen peroxide removal, humans with genetic deficiency of catalase - "acatalasemia" — or mice genetically engineered to lack catalase completely, suffer few ill effects.

\section{Estimation of brain reduced glutathione (gsh) activity}

Glutathione is a cysteine-containing peptide found in most forms of aerobic life. It is not required in the diet and is instead synthesized in cells from its constituent amino acids. Glutathione has antioxidant properties since the thiol group in its cysteine moiety is a reducing agent and can be reversibly oxidized and reduced. In cells, glutathione is maintained in the reduced form by the enzyme glutathione reductase and in turn reduces other metabolites and enzyme systems, such as ascorbate in the glutathione-ascorbate cycle, glutathione peroxidases and glutaredoxins, as well as reacting directly with oxidants 8.Due to its high concentration and its central role in maintaining the cell's redox state, glutathione is one of the most important cellular antioxidants. ${ }^{18-}$

${ }^{19}$ Reduced Glutathione estimation in brain homogenate is measured according to the Ellman method. This method is based on the development of a yellow colour when 5, 5'-dithio- bis-2-nitrobenzoic acid (DTNB) is added to the compound containing the sulfhydryl groups.

\section{Statistical Analysis:}

All the values of the experimental results were expressed as mean \pm standard error of mean (SEM). One-way ANOVA followed by Dunnet test. Graph Pad Prism (version 5.03) software was used for all statistical analysis and $\mathrm{p}<0.05$ was considered as significant.

\section{Results and Discussion:}

Phytochemical studies of Fraction A(Hexane: Ethyl acetate)revealed the Fraction presence of fatty acids, phenolics and flavonoids.Table.No:2

Table.No:2: Identification of phytochemical constituents:

\begin{tabular}{|l|l|l|l|l|l|l|l|}
\hline S.NO & $\begin{array}{l}\text { Phytochemical } \\
\text { tests }\end{array}$ & H:EA (95 : 5) & H:EA (70 30) & H:EA (50 : 50) & EA only & H only & M only \\
\hline 1 & Alkaloids & - & & & - & & \\
\hline 2 & Glycosides & - & & & - & & \\
\hline 3 & Tannins & - & & & - & & - \\
\hline 4 & Phenols & ++ & - & & - & \\
\hline 5 & Flavonoids & ++ & ++ & & - & & - \\
\hline 6 & Saponins & - & - & & - & & - \\
\hline 7 & Steroids & ++ & ++ & & & & \\
\hline
\end{tabular}

Where (+) Indicates presence, and (-) Indicates absence

\section{H- Hexane,EA-Ethyl acetate,M-methanol}



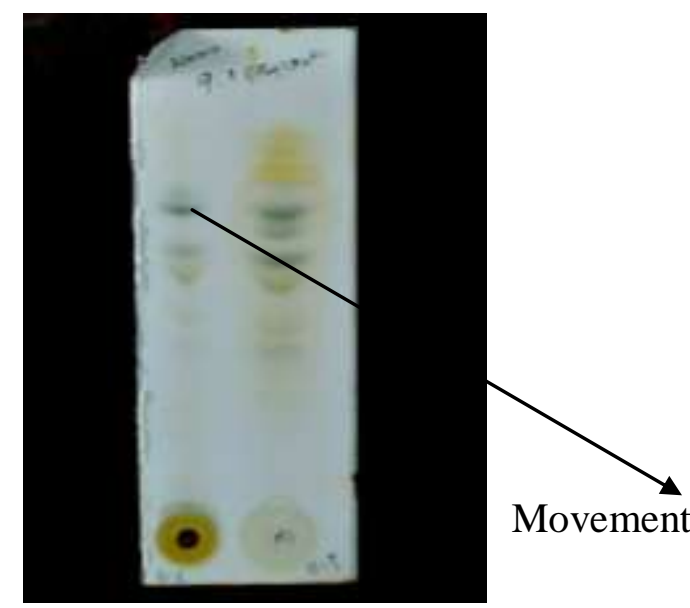

Movement of Hexane: Ethyl acetate(95:5)

Fraction

Figure:2: Thin Layer chromatography studies:

Acute oral toxicity studies:

No abnormal behaviour and no mortality observed for HEMBR

Table:3: Estimation of percentage inhibition of DPPH scavenging activity

\begin{tabular}{|c|c|c|c|}
\hline S.no & CONC.( $\boldsymbol{\mu g})$ & $\begin{array}{l}\text { \% INHIBITION OF } \\
(\mathbf{A A})\end{array}$ & H:EA(5:95 ratio) \\
\hline 1 & 50 & 46.57 & 45.77 \\
\hline 2 & 100 & 55.64 & 49.33 \\
\hline 3 & 150 & 63.82 & 60 \\
\hline 4 & 200 & 65.94 & 61.7 \\
\hline 5 & 250 & 65.86 & 64.44 \\
\hline 6 & IC 50 & 65 & 84 \\
\hline
\end{tabular}

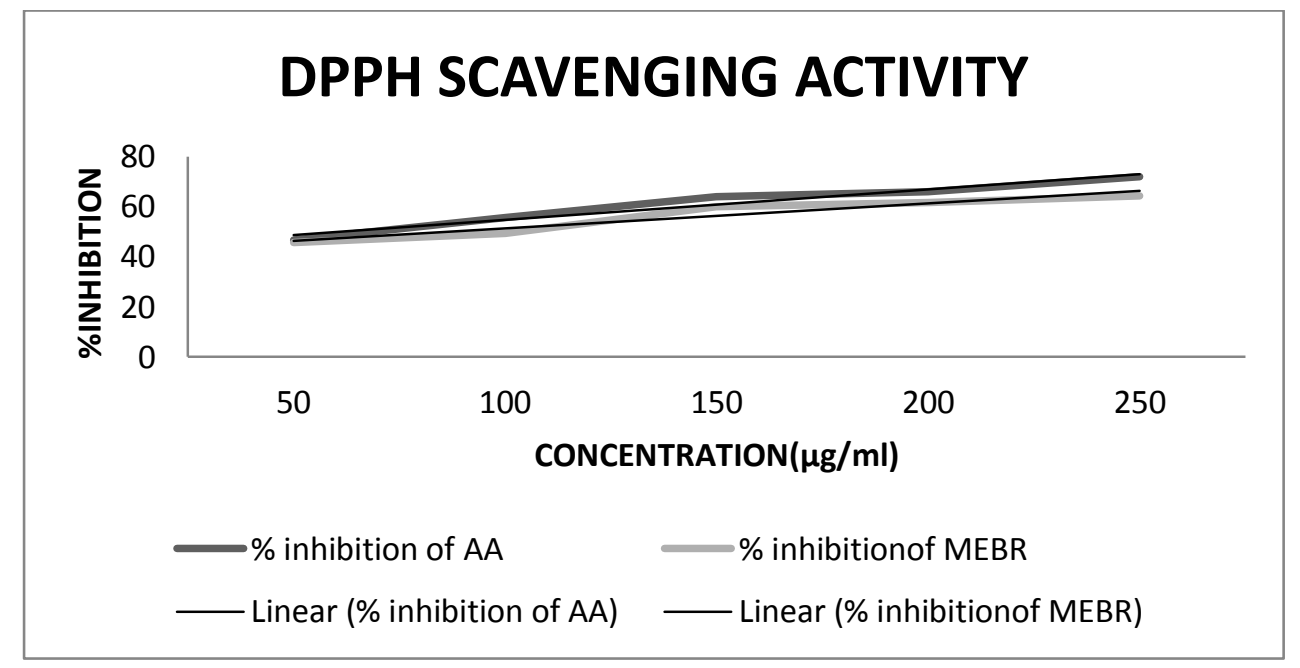

Figure:3 : Percentage inhibition of DPPH scavenging activity 


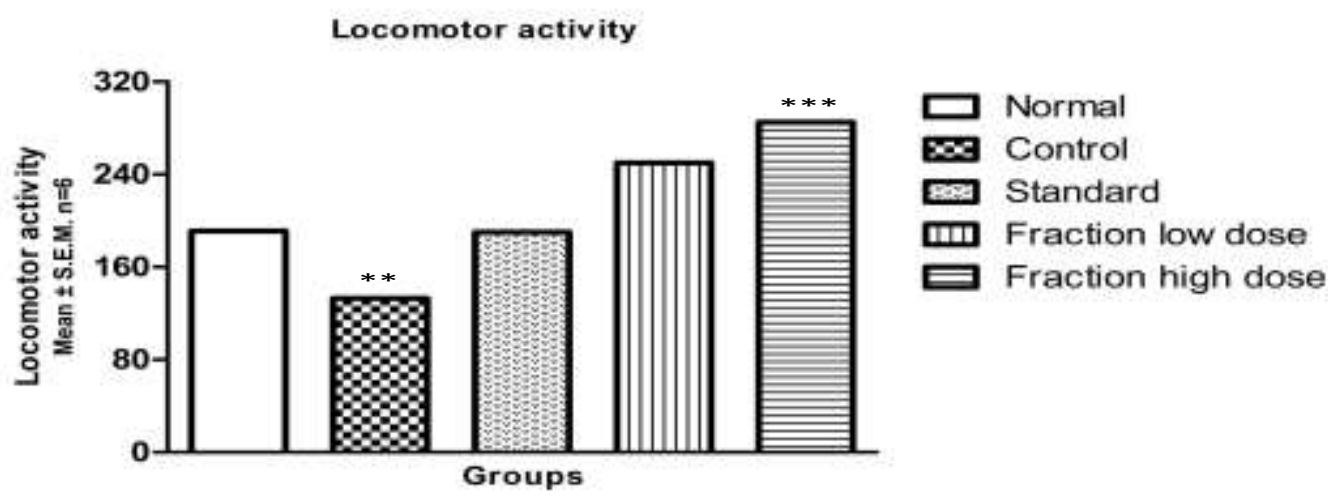

Figure:4: Effect of MEBR fraction A on Locomotor activity. All values are expressed in mean \pm SEM. Scopolamine treated group animals show decrease in locomotor response when compared to normal control group of animals, where as Donepzil treated animals increase in locomotor response when compared to scopolamine treated animals and MEBR fraction $A(100 \mathrm{mg} / \mathrm{kg})$ treated animals significantly decrease in active avoidance response $(* * * p<0.001)$ compared to scopolamine treated animals.

Spatial learning by Elevated plus mazes

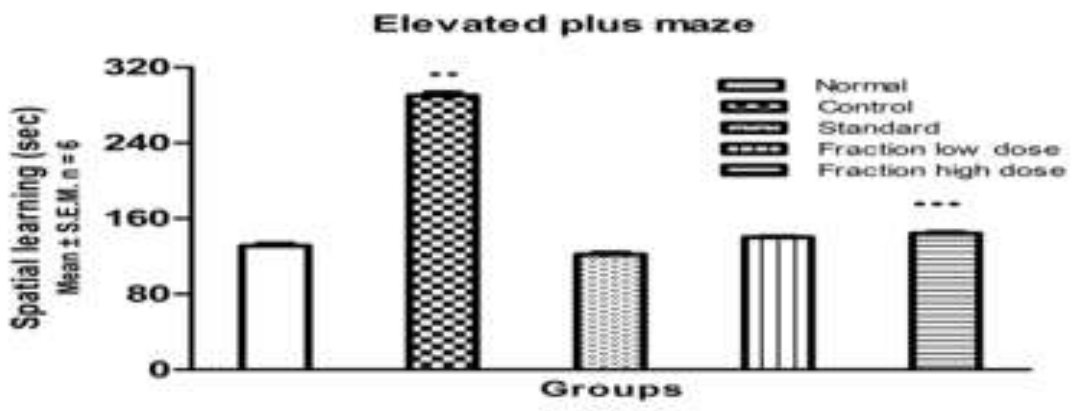

Figure: 5: Effect of MEBR fraction A on passive avoidance response. All values are expressed in mean \pm SEM. Scopolamine treated group animals show increase in active avoidance response when compared to normal control group of animals, where asDonepzil treated animals decrease in active avoidance response when compared to scopolamine treated animals and MEBR fraction $A(100 \mathrm{mg} / \mathrm{kg})$ treated animals significantly decrease in active avoidance response $(* * * \mathbf{p}<0.001)$ compared to scopolamine treated animals. 
Escape latency by Morris water maze

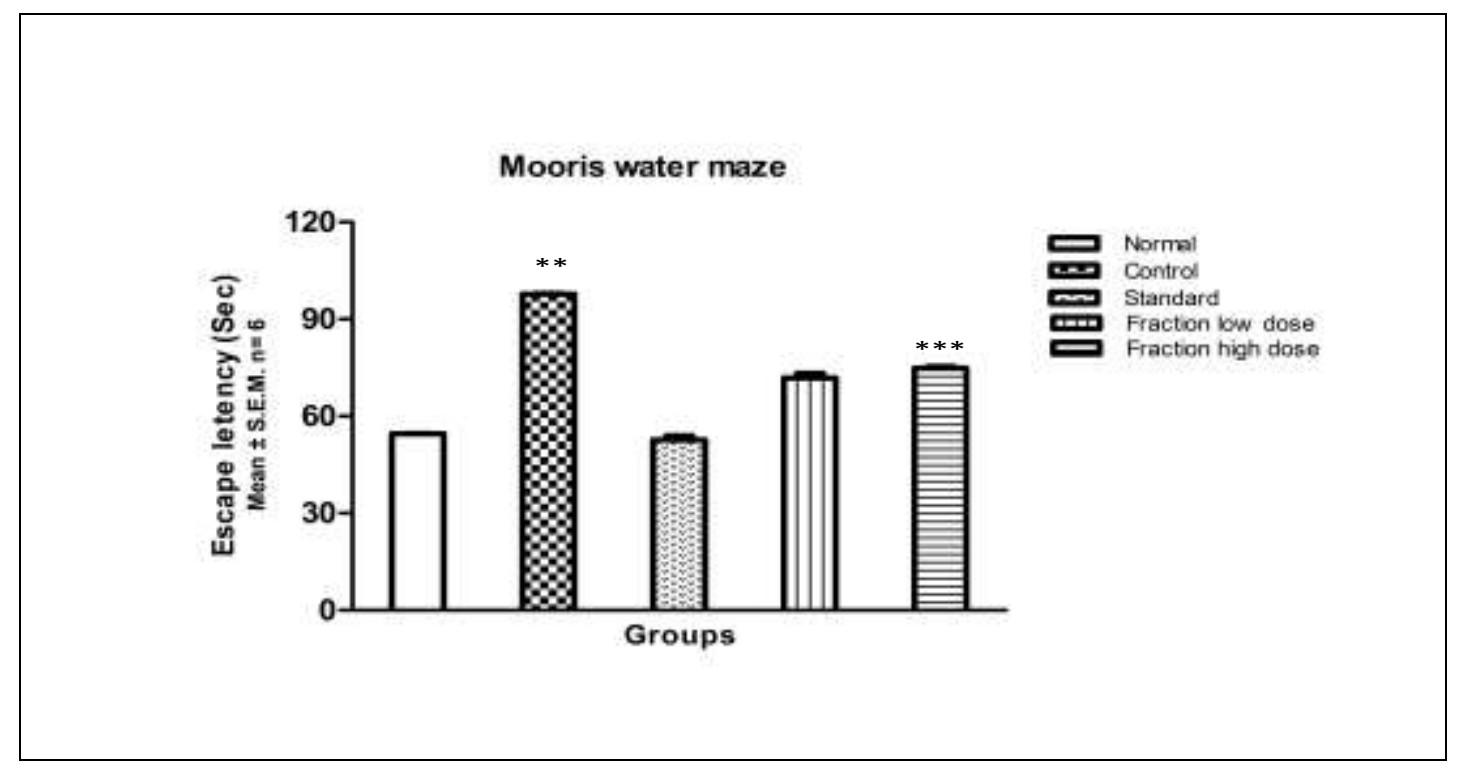

Figure: 06 : Effect of MEBR fraction A on escape latency. All values are expressed in mean \pm SEM. Scopolamine treated group animals show increase in escape latency time when compared to normal control group of animals, whereas Donepzil treated animals decrease in escape latency tim when compared to scopolamine treated animals and MEBR fraction $A(100 \mathrm{mg} / \mathrm{kg})$ treated animals significantly decrease in escape latency time $(* * * p<0.001)$ compared to scopolamine treated animals.

Acetyl Cholinesterase: (u/mg/protein)

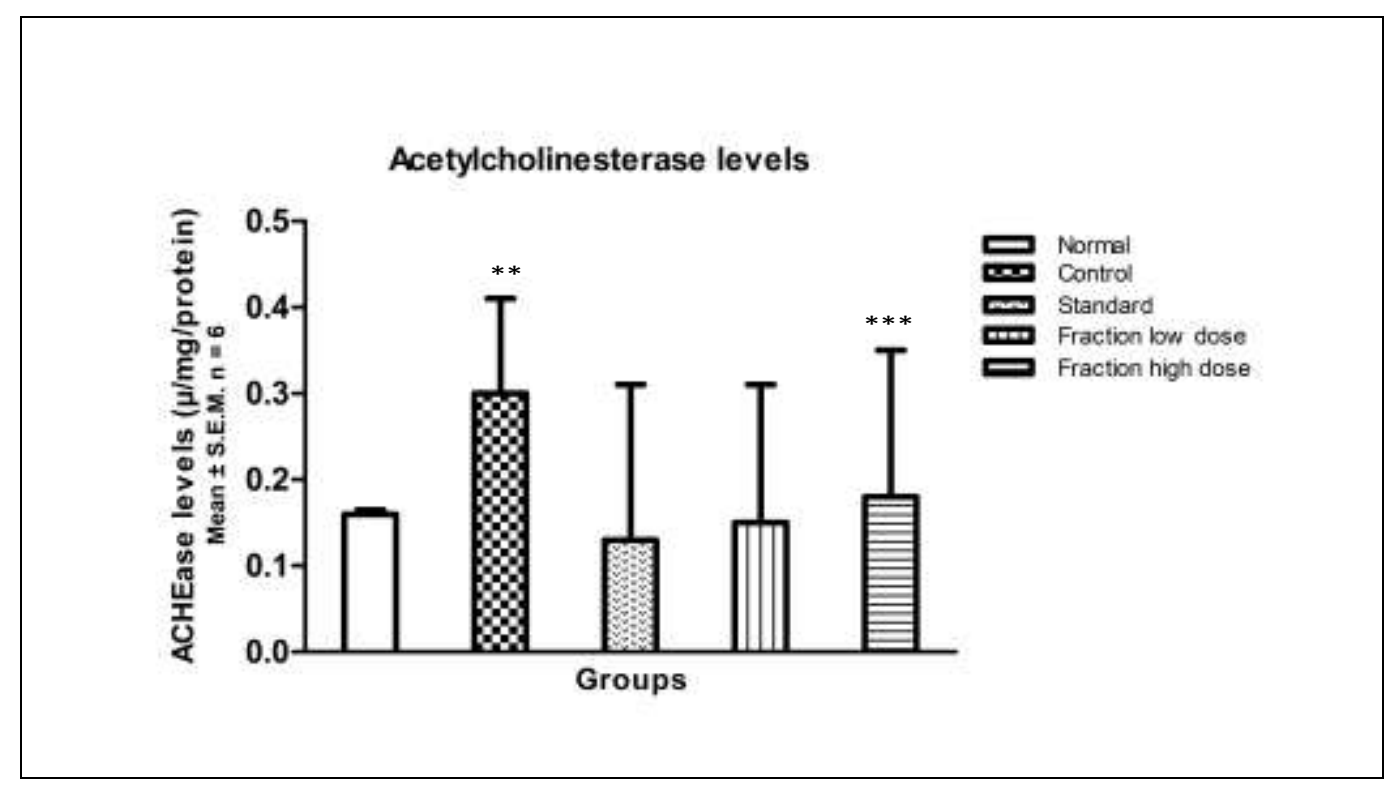

Figure7:Effect of MEBR fraction A on acetyl cholinesterase. All values are expressed in mean \pm SEM. Scopolamine treated group animals show increase in acetyl cholinesterase levels when compared to normal control group of animals, whereas Donepzil treated animals decrease in acetyl cholinesterase levels when compared to scopolamine treated animals and MEBR fraction A treated animals significantly decrease in acetyl cholinesterase levels $(* * * \mathrm{p}<0.001)$ compared to scopolamine treated animals. 


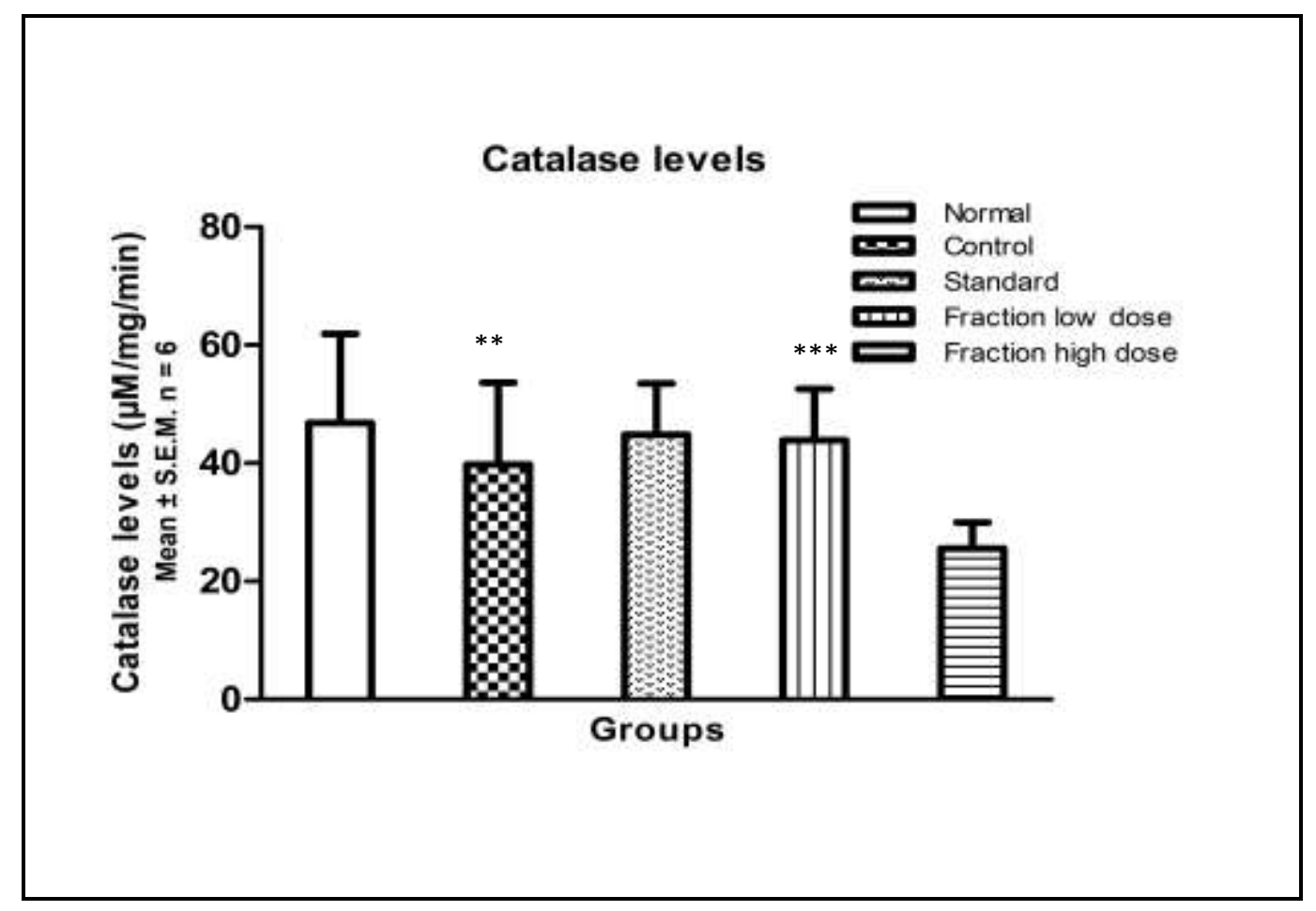

Figure 08:Effect of MEBR fraction A on catalase. All values are expressed in mean \pm SEM. Scopolamine treated group animals show decrease in catalase levels when compared to normal control group of animals, whereas Donepzil treated animals decrease in catalase levels when compared to scopolamine treated animals and MEBR fraction $\mathrm{A}(100 \mathrm{Mg} / \mathrm{Kg})$ treated animals significantly increase in catalase levels $(* * * \mathbf{p}<0.001)$ compared to scopolamine treated animals

Reduced Glutathione levels $(\mu \mathrm{mol} / \mathrm{g}$ tissue)

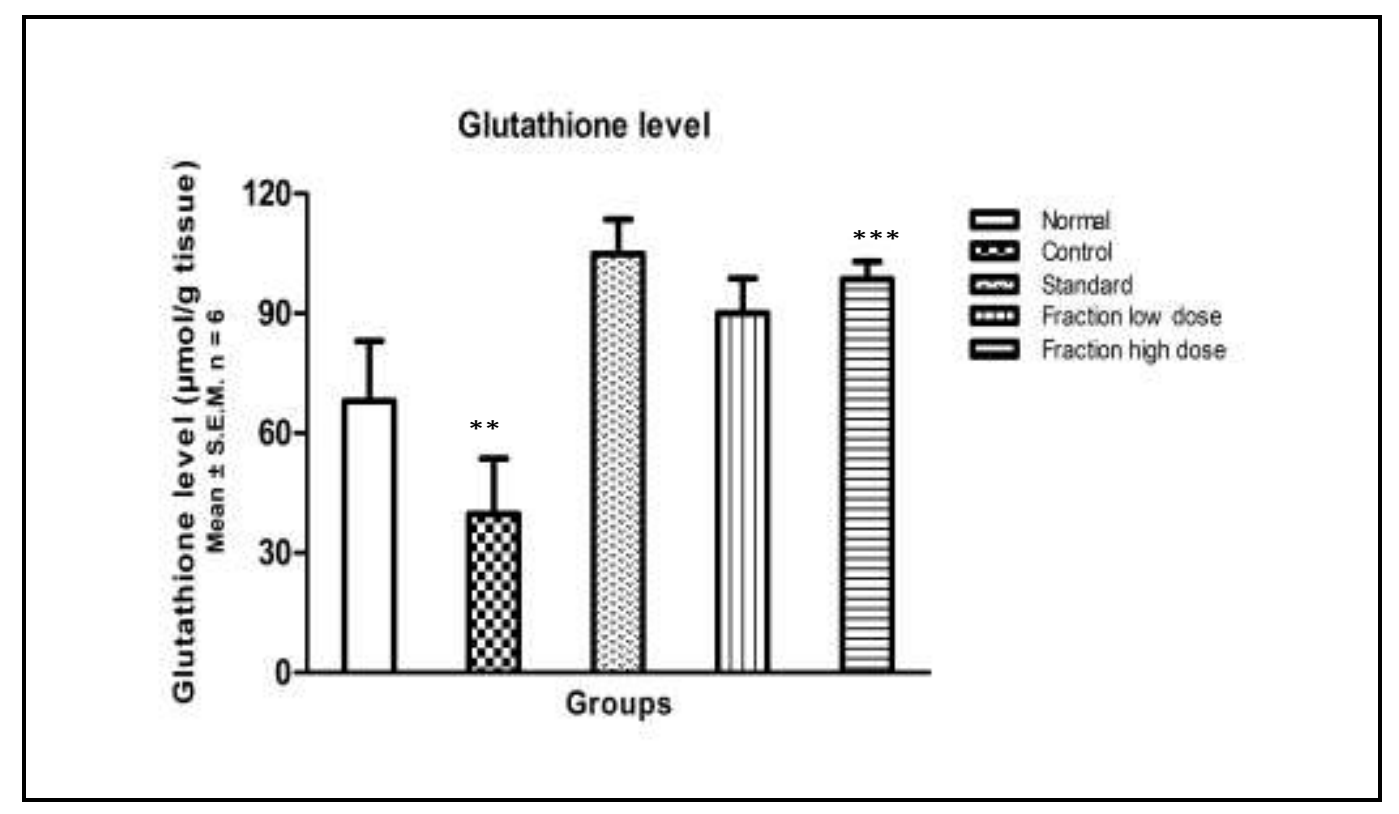

Figure 09: Effect of MEBR fraction $A$ on glutathione. All values are expressed in mean \pm SEM. Scopolamine treated group animals show decrease in glutathione levels when compared to normal control group of animals, whereas Donepzil treated animals increase in glutathione levels when compared to scopolamine treated animals and MEBR fraction $A(100 \mathrm{Mg} / \mathrm{Kg})$ treated animals significantly increase in glutathione levels $(* * * \mathbf{p}<0.001)$ compared to scopolamine treated animals. 


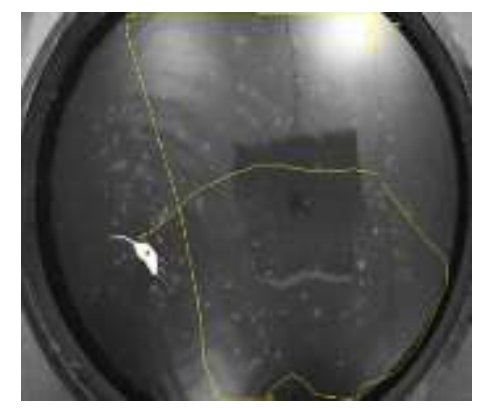

Group I Control

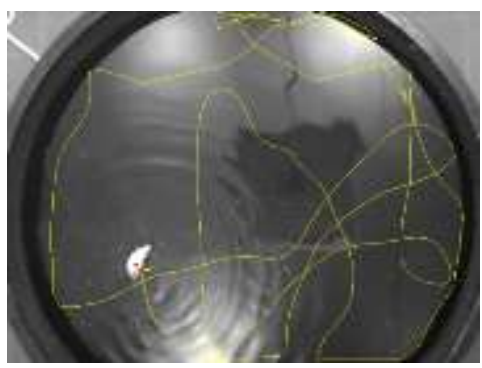

Group IV MEBR Fraction A 50mg

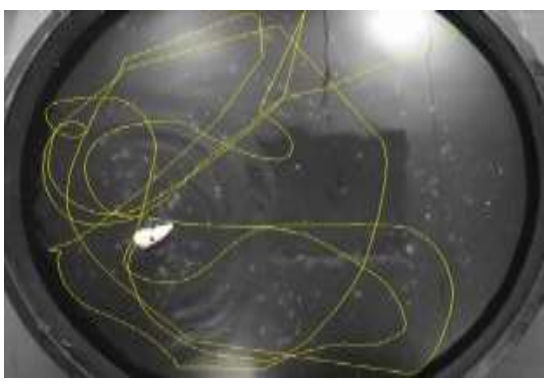

Group II Toxic Control

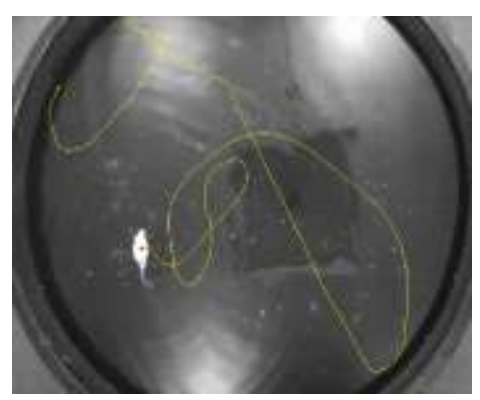

Group III Standard

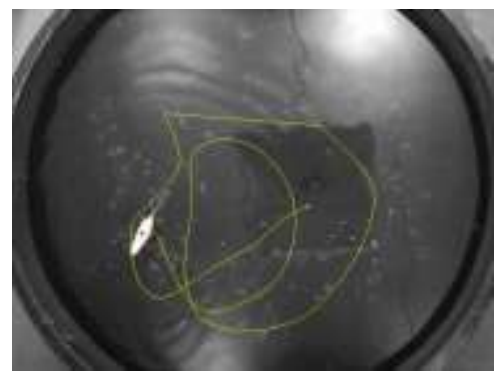

Group V MEBR Fraction A 100mg

Figure 10: Video tracking for Escape latency time by Morris water Maze:
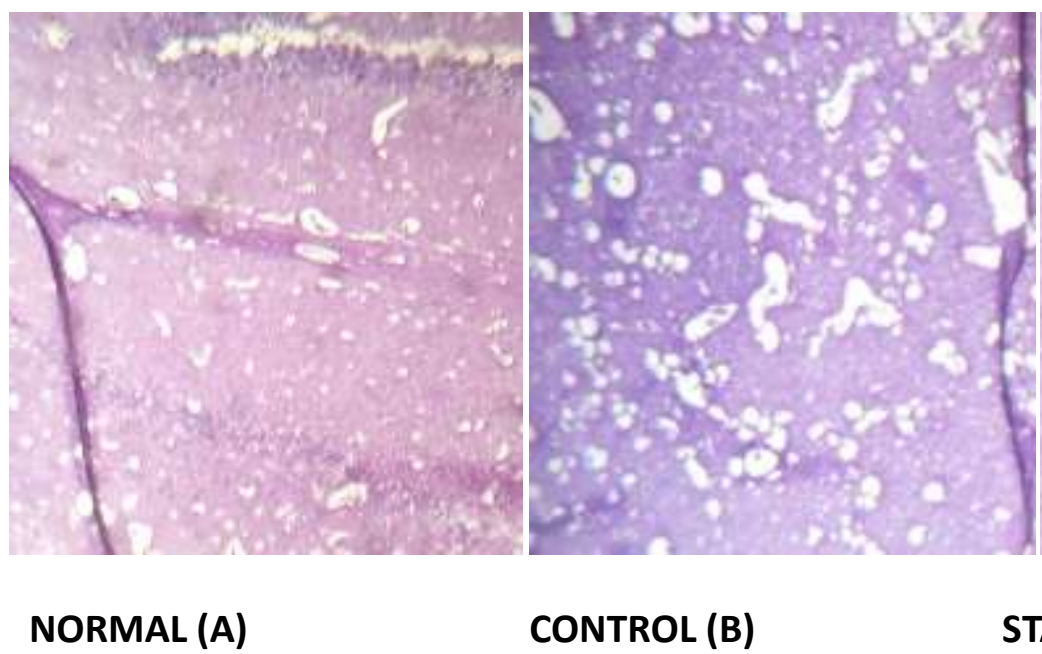

STANDARD (C)

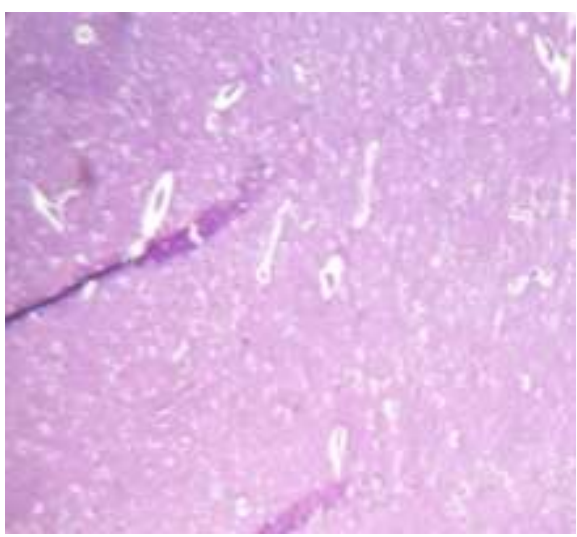

FRACTION LOW DOSE (D)

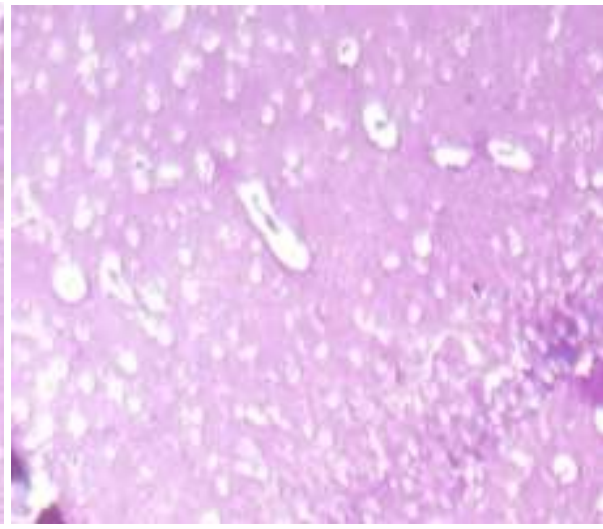

FRACTION HIGH DOSE (E)

Figure 11: Histopathological studies 
A. Normal control rat showing normal histological structure of Hippocampus

$>$ B.Scopolamine induced dementia rat showing severe congestion of blood capillaries with perivascular edema (Scars) together with edema and amyloid plaques in hippocampus.

$>$ C. Scopolamine induce dementia rat treated with donepezil showing diffuse gliosis and shrinkage of nuclei in pyramidal cell in hippocampus.

$>$ D. When compared with control, fraction treated rat show less formation of amyloid plaques in hippocampus region of brain

$>$ E.When compared with control, fraction high dose treated rat show less formation of amyloid plaques in hippocampus region of brain

\section{Discussion:}

Medicinal plants are store house of phytochemicals for treatment of countless major and minor disease. In the treatment of the neurodegenerative particularly $\mathrm{AD}$ the phyto constituents of medicinal plants play a crucial role as stated earlier. The scopolamine-induced animal model of dementia and oxidative stress is widely used as a primary screening test for the determination of anti-Alzheimer effect of unknown plants or drugs. Scopolamine is widely used as animal AD model for screening anti-Alzheimer's disease ${ }^{20}$. Scopolamine acts as muscarinic cholinergic antagonist cause impairment in cognition and learning ${ }^{21}$. Scopolamine amnesia in animal model of Alzheimer's is associated with increased level oxidative stress observed in the brain.

Phytochemical analysis showed the presence of lower proportions of phenols and flavonoids which are responsible for anti-Alzheimer activity and also showed high proportions of linoleic acid (fatty acids).Table no:2

The Morris water maze model was used as a Behavioural model to assess the effect of the extract on memory. The Morris water maze model is widely used to assess the effects of drugs on learning and memory. In this model, a decrease in escape latency (EL) and an increase in time spent in the target quadrant (TSTQ) indicate improvement of learning and memory. The MESL showed a significant decrease in EL and a significant increase in TSTQ in Scopolamine mice.

Spatial long term memory is evaluated by EPM test in which the measured parameters were ITL and RTL. The time spent by the rat to move from the open arm to the closed arm in this test was recorded as ITL. After $24 \mathrm{hrs}$ of ITL the retention of learned task was studied as RTL. In this test, a decrease in RTL on 7th day after the ILT on 6th day respectively indicated improvement of spatial long-term memory of rats as compared to disease control and control group.

The results of the Actophotometer show that there was a significant decrease in locomotor activity (EL) of Group II (Alzheimer group) rats when compared to Group I (Normal control) rats. However, the time taken by the rat to locomotor activity increased significantly in Group III (Treatment group; $250 \mathrm{mg} / \mathrm{kg}$ ) and Group IV (Treatment group; $500 \mathrm{mg} / \mathrm{kg}$ ) rats when compared to Group II (Alzheimer group) rats.

To find out oxidative stress at various levels the defensive antioxidant enzymes in rat brain were measured. DPPH scavenging activity of plant extract (IC50 84) showed the IC50 value close to the ascorbic acid (IC50 65). CAT, which is present virtually in all mammalian cells, is responsible for the removal of $\mathrm{H}_{2} \mathrm{O}_{2}$. Therefore, one of the oxidative stress indices was estimated in rat brain that is CAT. There was a significant reduction in antioxidant defensive enzyme CAT content observed in scopalamine induced rats. The levels of CAT were significantly restored by treatment with MEBR., Biophytum reinwardtii reduced oxidative stress in scopolamine-induced stress in rat brain suggesting the antioxidant activity of the plant. The neuroprotective activity of the plant may be due to its antioxidant property, which reinforces Anti-Alzheimers activity of Biophytum reinwardtii.

Scopolamine treatment significantly increased the brain AchE level compared to control group (Figure 5). Standard drug (donepezil) and test drugs(H:EA) treatment significantly inhibited the brain AchE level compared to their corresponding scopolaminetreated groups.

Glutathione reduced, glutathione reductase and glutathione-S-transferase is thought to be the fundamental antioxidant enzymes, for they are closely related to the direct elimination of reactive oxygen species. Therefore, the reduction in the activity of these enzymes may result in a number of deleterious effects 
due to the accumulation of superoxide radicals and hydrogen peroxide, linked with neurodegenerative disease. In this study reduced glutathione levels are decreased in scopolamine treated group and increased levels is observed in treatment groups.

Normal control rat showing normal histological structure of Hippocampus, Scopolamine induced dementia rat showing severe congestion of blood capillaries with perivascular edema (Scars) together with edema and amyloid plaques in hippocampus. Scopolamine induce dementia rat treated with donepezil showing diffuse gliosis and shrinkage of nuclei in pyramidal cell in hippocampus. When compared with control, fraction treated rat show less formation of amyloid plaques in hippocampus region of brain. When compared with control, fraction high dose treated rat show less formation of amyloid plaques in hippocampus region of brain. When compared with control, crude low dose treated rat show less formation of amyloid plaques in hippocampus region of brain. When compared with control, crude high dose treated rat show less formation of amyloid plaques in hippocampus region of brain. When comparing fraction and crude extract treated groups fraction treated group shows better result.

\section{Conclusion:}

The present study clearly demonstrates that MEBR fraction A contain phenols, flavonoids. MEBR fraction $(50$ and $100 \mathrm{mg} / \mathrm{kg}$ ) significantly attenuate scopolamine-induced dementia by improving the learning, memory, antioxidant potentiality and anti-cholinesterase activity. Therefore, this Fraction can be a potential novel therapeutic strategy for controlling neurodegenerative dementia especially AD. Further advance studies are needed to expose the possible mechanism of action.

\section{Acknowledgment:}

Authors are thankful to management of Chebrolu Hanumaiah Institute of Pharmaceutical Sciences, Guntur and Shri Vishnu College of Pharmacy, Bhimavaram for providing facilities to conducting this work.

\section{References:}

1. 2010Alzheimer's disease facts and figures (2010) Alzheimers Dement 6, 158194.

2. Sorensen AA (2009) Alzheimer's disease research: scientific productivity and impact of the top 100 investigators in the field. J Alzheimers Dis 16, 451465.

3. Braak H, Braak E, Bohl J (1993) Staging of Alzheimer related cortical destruction. EurNeurol33, 403408.

4. Wenk GL. Neuropathologic Changes in Alzheimer's Disease. The Journal of Clinical Psychiatry. 2003; 64 Suppl 9:7-10. PMID 12934968.

5. Where, when, and in what form does sporadic Alzheimer's disease begin?. Current Opinion in Neurology. December 2012; 25(Pt 6):708-14.

6. Automated MRI Measures Identify Individuals with Mild Cognitive Impairment and Alzheimer's Disease. Brain. August 2009;132(Pt 8):2048-57.

7. Moan R. MRI Software Accurately IDs Preclinical Alzheimer's Disease. Diagnostic Imaging. 20 July 2009.

8. The Importance of Neuritic Plaques and Tangles to the Development and Evolution of AD. Neurology. 2004;62(11):1984-89.

9. Regional Distribution of Neurofibrillary Tangles and Senile Plaques in the Cerebral Cortex of Elderly Patients: A Quantitative Evaluation of a One-year Autopsy Population from a Geriatric Hospital. Cerebral Cortex. 1994;4(2):138-50.

10. Neurotrophic factors in Alzheimer's disease: role of axonal transport. Genes, Brain, and Behavior. 2008;7(Suppl 1):43-56.

11. Voisin T, Vellas B. Diagnosis and treatment of patients with severe Alzheimer's disease. Drugs Aging 2009;26(2):135-44.

12. Fitzpatrick AL, Kuller LH, Ives DG, Lopez OL, Jagust W, Breitner JC, et al. Incidence and prevalence of dementia in the Cardiovascular Health Study. J Am GeriatrSoc 2004;52(2):195-204.

13. Kukull WA, Higdon R, Bowen JD, McCormick WC, Teri L,Schellenberg GD, et al. Dementia and Alzheimer disease incidence: A prospective cohort study. Arch Neurol 2002;59(11):1737-46. 
14. Evans DA, Bennett DA, Wilson RS, Bienias JL, Morris MC,Scherr PA, et al. Incidence of Alzheimer disease in a biracial urban community: Relation to apolipoprotein E allele status. ArchNeurol 2003;60(2):185-9.

15. Stern Y, Gurland B, Tatemichi TK, Tang MX, Wilder D, Mayeux R. Influence of education and occupation on the incidence of Alzheimer's disease. JAMA 1994;271(13):1004-10.

16. Evans DA, Hebert LE, Beckett LA, Scherr PA, Albert MS, Chown MJ, et al. Education and other measures of socioeconomic status and risk of incident Alzheimer disease in a defined population of older persons. Arch Neurol 1997;54(11):1399-405.

17. Dementia and Chronic Cognitive Impairment; Rehabilitation and Care. National Institute for Health Research. Available athttp://dementia.cochrane.org/our-reviews. Accessed October 21, 2013.

18. Olazarán J, Reisberg B, Clare L, Cruz I, Peña-Casanova J, del Ser T, et al. Nonpharmacological therapies in Alzheimer'sdisease: A systematic review of efficacy. Dement GeriatrCognDisord 2010:30:161-78.

19. Brodaty H, Arasaratnam C. Meta-analysis of nonpharmacological interventions for neuropsychiatric symptoms of dementia. Am J Psychiatry 2012:169:946-53.

20. Hebert LE, Weuve J, Scherr PA, Evans DA.Alzheimer disease.

21. Reiman EM, Quiroz YT, Fleisher AS, Chen K, Velez-Pardos C, Jimenez-Del-Rio M, et al. Brain imaging and fluid biomarker analysis in young adults at genetic risk for autosomal dominant Alzheimer's disease in the presenilin 1 E280A kindred: A case-control study. Lancet Neurology 2012;11(2):1048-56. 